\title{
A Non-Stationary Wideband MIMO Channel Model for High-Mobility Intelligent Transportation Systems
}

\author{
Ammar Ghazal, Cheng-Xiang Wang, Senior Member, IEEE, Bo Ai, Senior Member, IEEE, \\ Dongfeng Yuan, Senior Member, IEEE, and Harald Haas, Member, IEEE
}

\begin{abstract}
The recent development of high-speed trains (HSTs), as a high-mobility intelligent transportation system (ITS), and the growing demands of broadband services for HST users introduce new challenges to wireless communication systems for HSTs. The deployment of mobile relay stations (MRSs) on top of the train carriages is one of the promising solutions for HST wireless systems. For a proper design and evaluation of HST wireless communication systems, we need accurate channel models that can mimic the underlying channel characteristics for different HST scenarios. In this paper, a novel non-stationary geometry-based stochastic model (GBSM) is proposed for wideband multipleinput multiple-output (MIMO) HST channels in rural macrocell (RMa) scenarios. The corresponding simulation model is then developed with angle parameters calculated by the modified method of equal areas (MMEA). Both channel models can also be used to model non-stationary V2I channels in vehicular communication networks. The system functions and statistical properties of the proposed channel models are investigated based on a theoretical framework that describes non-stationary channels. Numerical and simulation results demonstrate that the proposed channel models have the capability to characterize the non-stationarity of HST channels. The statistical properties of the simulation model, verified by the simulation results, can match those of the proposed theoretical GBSM. An excellent agreement is achieved between the stationary intervals of the proposed simulation model and those of relevant measurement data, demonstrating the utility of the proposed channel models.
\end{abstract}

Index Terms - High-speed train (HST) channels, vehicle-toinfrastructure (V2I) channels, GBSM, non-stationary MIMO channel models, statistical properties.

Mauscript received October 03, 2013; revised February 14, 2014 and June 06, 2014; accepted July 21, 2014. The Associate Editor for this paper was F. Wang.

The authors would like to acknowledge the support from the Opening Project of the Key Laboratory of Cognitive Radio and Information Processing (Guilin University of Electronic Technology), Ministry of Education (Grant No. 2013KF01), State Key Laboratory of Rail Traffic Control and Safety (Contract No. RCS2012K003), Beijing Jiaotong University, the National Natural Science Foundation of China (Grant No. 61222105), Beijing Municipal Natural Science Foundation (Grant No. 4112048), and 863 National Project (Grant No. 2014AA01A706).

A. Ghazal and C.-X. Wang are with the Joint Research Institute for Signal and Image Processing, School of Engineering and Physical Sciences, Heriot-Watt University, Edinburgh, EH14 4AS, UK (e-mail: \{ag289, chengxiang.wang\}@hw.ac.uk). C.-X. Wang is the corresponding author.

B. $\mathrm{Ai}$ is with the State Key Laboratory of Rail Traffic Control and Safety, Beijing Jiaotong University, Beijing, 100044, China (e-mail: boai@bjtu.edu.cn).

D. Yuan is with the School of Information Science and Engineering, Shandong University, Jinan, 250100, China (e-mail: dfyuan@sdu.edu.cn).

H. Haas is with the Joint Research Institute for Signal and Image Processing, Institute for Digital Communications, University of Edinburgh, Edinburgh, EH9 3JL, U.K. (e-mail: h.haas@ed.ac.uk).

\section{INTRODUCTION}

Intelligent transportation systems (ITSs) consider all types of communications between vehicles, i.e., vehicle-to-vehicle $(\mathrm{V} 2 \mathrm{~V})$, vehicle-to-roadside (V2R) or vehicle-to-infrastructure (V2I), and information and communication technologies for rail, water, and air transport [1], [2]. As a fast and convenient ITS, railways that operate trains with a high speed of more than $300 \mathrm{~km} / \mathrm{h}$ have attracted more and more attentions recently. With the increase of train speeds, wireless communication systems face various challenges such as fast handover [3], high penetration losses, limited visibility in tunnels, large Doppler, delay, and angular spreads. The widely used Global System for Mobile Communication Railway (GSM-R) can only provide a data rate of up to $200 \mathrm{kbps}$ [4], besides the fact that GSM-R is mainly used for train control rather than providing communications for train passengers. Therefore, GSM-R cannot meet the requirements for future high data rate transmissions. International Union of Railways has recommended that GSM$\mathrm{R}$ has to be replaced by long-term evolution-Railway (LTE$\mathrm{R})$, which is a broadband railway wireless communication system based on LTE-Advanced (LTE-A) [5]. However, both systems still adopt conventional cellular architecture where mobile stations (MSs) inside trains communicate directly with outdoor base stations (BSs). Such an architecture leads to a spotty coverage and high penetration losses of wireless signals traveling into the carriages of high-speed trains (HSTs) [6]. The received signals at the MS on board will experience fast changing channels resulting in high signaling overhead and high possibility of drop calls and handover failure.

The above problems can be mitigated by deploying other cellular architectures, such as distributed antenna systems (DAS) [7], coordinated multipoint (CoMP) [8], and mobile relay station (MRS) (or Mobile Femtocell) [9]. In this paper, we will only consider using the promising MRS technology, as this has been adopted by IMT-Advanced (IMT-A) [10] and WINNER II [11] systems. This is performed by deploying dedicated MRSs on the surface of the train to extend the coverage of the outdoor BS into train carriages. As a result, the effect of frequent handover will be significantly reduced by performing a group handover with the MRS instead of dealing with the individual handover of each passenger [12]. By considering MRS solution, we will have two channels: an outdoor channel between the BS and MRS and an indoor one between the MRS and MS. Radio channels between the 
MRS and MSs in the carriages resemble indoor environments and hence can be modeled using the existing indoor channel models [13]. Here, we will focus on the outdoor channel because of the research challenges due to the high velocity of the MRS. This outdoor channel is very similar to a V2I communication channel.

Demonstrating the feasibility of wireless systems in HST scenarios before implementation is not possible without accurate channel models that are able to mimic key characteristics of HST wireless channels, such as the non-stationarity. Similar to V2V channels [14]-[18], the non-stationarity of the HST channels means that the channel statistics can change rapidly over a short period of time. Several measurement campaigns [19]-[24] for different HST environments (rural macro-cell (RMa), tunnels, viaducts, hilly terrains, and U-shaped) were conducted but they mainly focused on large-scale fading parameters, such as path loss and delay spread, and thus ignored small-scale fading parameters.

Channel models in the literature have also failed to demonstrate different propagation parameters of wireless channels in HST scenarios. Adopting a conventional cellular architecture, the LTE-A system [5] provided a relatively simple singlepath channel model that supports two scenarios, i.e., open space and tunnels, but ignores the non-stationarity of HST channels. In [25], the propagation channels between HSTs and fixed BSs were modeled using the ray-tracing method, which incorporates a detailed simulation of the actual physical wave propagation process based on an approximation to Maxwell equations [26]. However, the implementation of ray-tracing models always requires extensive computational resources. In both the RMa scenario in WINNER II [11] and moving networks scenario in IMT-A channel models [10], the train speed can be up to $350 \mathrm{~km} / \mathrm{h}$ and the MRS technology is employed. Both channel models introduced time evolution concept to explicitly simulate the non-stationarity of channels. However, it has been demonstrated in [27] how the stationary interval, defined as the maximum time duration over which the channel satisfies the wide-sense stationary (WSS) condition, of these two standard channel models is considerably longer than that of real HST channels. For a train speed of $324 \mathrm{~km} / \mathrm{h}$, the reported stationary intervals of the standardized models and the measured HST channel were $37.8 \mathrm{~ms}$ and $20 \mathrm{~ms}$, respectively [27]. Consequently, the stationary distance of the standardized channel models is equal to $3.4 \mathrm{~m}$, while it is only $1.8 \mathrm{~m}$ for the measured HST channel. The statistical characterization of WSS multiple-input multiple-output (MIMO) channels has been investigated extensively in the literature [28]-[30]. In contrast, only few papers [31]-[35] have studied the statistical properties of non-stationary channels.

To fill the above research gaps, it is highly desirable to design accurate channel models that consider the nonstationarity of HST scenarios by taking into account timevarying small-scale fading parameters, like angles of arrival (AoAs) and angles of departure (AoDs). Also, it is essential to establish a theoretical framework that characterizes nonstationary mobile fading channels in terms of their system functions and correlation functions (CFs). The major contributions and novelties of this paper are summarized as follows.
1) We study the system functions and CFs of non-stationary channel models and propose a theoretical framework for deriving the corresponding statistical properties.

2) By considering the deployment of MRS in HST communication systems, we propose a theoretical geometrybased stochastic model (GBSM) for wideband nonstationary MIMO HST channels in RMa scenarios. The proposed model has time-varying angular parameters and time-varying distance between the transmitter (Tx) and receiver $(\mathrm{Rx})$. Then, we derive and study its timevariant space-time (ST) CF, time-variant space-Doppler (SD) power spectrum density (PSD), and local scattering function (LSF).

3) The aforementioned theoretical (reference) model assumes an infinite number of effective scatterers and therefore cannot be used for simulations. Thus, we further develop a corresponding sum-of-sinusoids (SoS) based simulation model for wideband non-stationary MIMO HST channels with reasonable complexity, i.e., a finite number of sinusoids or effective scatterers. The angle parameters of the proposed simulation model are calculated by adopting the modified method of equal areas (MMEA). The relevant statistical properties of the developed simulation model are derived, verified by simulations, and compared with those of the proposed theoretical GBSM. Furthermore, the utility of the proposed simulation model is validated using measurement data.

The rest of this paper is organized as follows. The system functions of non-stationary channel models are presented in Section II. Section III proposes a theoretical non-stationary GBSM for wideband MIMO HST channels in RMa scenarios and studies its statistical properties. In Section IV, the SoS simulation model for wideband MIMO HST channels is proposed and its statistical properties are investigated. Numerical and simulation results are presented and analyzed in Section V. Finally, conclusions are drawn in Section VI.

\section{System FunCTIONS AND CFS OF NON-STATIONARY WIDEBAND MIMO CHANNELS}

In this section, we will derive the system functions and $\mathrm{CFs}$ that describe non-stationary wideband MIMO channels. The received signal experiencing non-stationary wideband mobile channels can be defined as a 3-dimensional (3D) stochastic process in terms of time $t$, delay $\tau$, and space $x$, which denotes the location of an antenna element in the antenna array in the $\mathrm{Tx} / \mathrm{Rx}$ [36]. It can be described by the spacetime-variant channel impulse response $h(t, \tau, x)$ [36], [37]. An alternative description of the non-stationary channel in space-time-frequency domain is the space-time-variant transfer function that can be obtained by taking the Fourier transform of $h(t, \tau, x)$ in terms of delay $\tau$, i.e.,

$$
L_{H}(t, f, x)=\int h(t, \tau, x) e^{-j 2 \pi f \tau} \mathrm{d} \tau .
$$

Other 3D system functions can be obtained by using Fourier transforms as in [36], [37]. The 6-dimensional (6D) spacetime-delay-variant space-time-delay CF can be obtained from 
the space-time-variant impulse response as follows:

$$
\begin{aligned}
R_{h}(t, \tau, x ; \Delta t, \Delta \tau, \Delta x) & =\mathrm{E}\{h(t, \tau, x) \\
& \left.\times h^{*}(t-\Delta t, \tau-\Delta \tau, x-\Delta x)\right\}
\end{aligned}
$$

where $\Delta t$ is the time lag (i.e., time difference), $\Delta \tau$ is the delay lag, and $\Delta x$ is the space lag (i.e., antenna element spacing [36]). Here, $(\cdot)^{*}$ denotes the complex conjugate operation and $\mathrm{E}\{\cdot\}$ designates the statistical expectation operator. Similarly, we can obtain the space-time-frequency-variant space-timefrequency $\mathrm{CF}$ as

$$
\begin{aligned}
R_{L}(t, f, x ; \Delta t, \Delta f, \Delta x) & =\mathrm{E}\left\{L_{H}(t, f, x)\right. \\
\times & \left.L_{H}^{*}(t-\Delta t, f-\Delta f, x-\Delta x)\right\}
\end{aligned}
$$

where $\Delta f$ is the frequency lag. The 6D CFs (2) and (3) are spatial extension of the 4D CFs proposed by Matz [32]. Matz also suggested another channel statistic for non-stationary channels, namely, the LSF $C_{H}(t, f, \tau, \nu)$, which describes the mean power of the effective scatterers causing delay-Doppler shifts $(\tau, \nu)$ at time $t$ and frequency $f$. Here, we extend the 4-dimensional (4D) LSF in [32] to the space and direction domains resulting in the $6 \mathrm{D} \operatorname{LSF} C_{H}(t, f, x, \tau, \nu, \Omega)$, which can be obtained as follows:

$$
\begin{aligned}
C_{H}(t, f, x, \tau, \nu, \Omega) & =\iiint R_{h}(t, \tau, x ; \Delta t, \Delta \tau, \Delta x) \\
& \times e^{-j 2 \pi(\nu \Delta t+f \Delta \tau+\Omega \Delta x)} \mathrm{d} \Delta t \mathrm{~d} \Delta \tau \mathrm{d} \Delta x \\
C_{H}(t, f, x, \tau, \nu, \Omega) & =\iiint R_{L}(t, f, x ; \Delta t, \Delta f, \Delta x) \\
& \times e^{-j 2 \pi(\nu \Delta t-\tau \Delta f+\Omega \Delta x)} \mathrm{d} \Delta t \mathrm{~d} \Delta f \mathrm{~d} \Delta x .
\end{aligned}
$$

Here, $\Omega$ is the direction of an antenna element in the antenna array in the Tx/Rx. The above general system functions and CFs of non-stationary channel models can be simplified by applying the following assumptions.

- Uncorrelated scattering (US): US means that different channel taps with different delays are uncorrelated. In [31], Bello showed that US channels are WSS in the frequency domain. Therefore, the 6D space-time-frequency-variant space-timefrequency $\mathrm{CF}$ in (3) will no longer depend on frequency $f$, i.e., it will be reduced to the 5-dimensional (5D) spacetime-variant space-time-frequency CF $R_{L}(t, x ; \Delta t, \Delta f, \Delta x)$. It follows that the $6 \mathrm{D}$ space-time-delay-variant space-timedelay $\mathrm{CF}$ in (2) will be reduced to the 5D space-time-delayvariant space-time $\mathrm{CF} R_{h}(t, \tau, x ; \Delta t, \Delta x)$.

- Antenna stationarity: Antenna stationarity means that the time, frequency, and antenna statistics, i.e., the correlation between different antenna elements separated by $\Delta x$, do not depend on the selected transmit or receive antennas [38]. It follows that the 6D space-time-frequency-variant spacetime-frequency $\mathrm{CF}$ in (3) will no longer depend on space $x$, i.e., it will be reduced to the 5D time-frequency-variant space-time-frequency CF $R_{L}(t, f ; \Delta t, \Delta f, \Delta x)$. Also the 6D space-time-delay-variant space-time-delay $\mathrm{CF}$ in (2) will be reduced to the $5 \mathrm{D}$ time-delay-variant space-time-delay $\mathrm{CF}$ $R_{h}(t, \tau ; \Delta t, \Delta \tau, \Delta x)$.

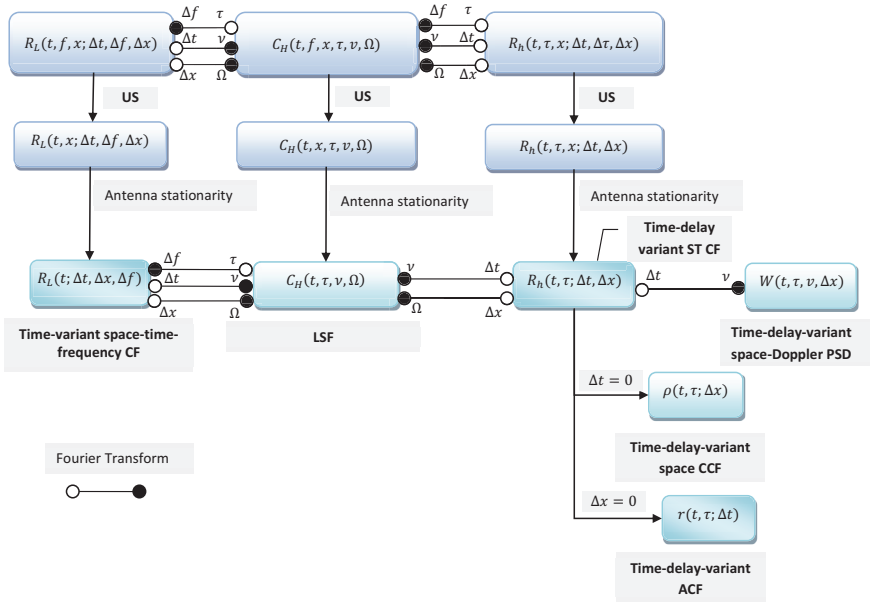

Fig. 1. Relationship of CFs for non-stationary HST channels.

In this paper, we assume that the channel satisfies both the US and antenna stationarity conditions. Antenna stationarity assumption is common for conventional MIMO channels using limited numbers of uniform linear array (ULA) antenna elements. However, this assumption becomes invalid in case of non-ULA antennas [38] and/or large MIMO channels with a large number (tens or even hundreds) of antenna elements [39]. By applying these two assumptions, the CFs will not depend on $f, \Delta \tau$, and $x$. Therefore, the $6 \mathrm{D}$ space-time-delay-variant space-time-delay CF in (2), space-time-frequency-variant space-time-frequency $\mathrm{CF}$ in (3), and the LSF in (5) will be reduced to 4-dimensional (4D) time-delay-variant space-time $\mathrm{CF} R_{h}(t, \tau, \Delta t, \Delta x)$, 4D timevariant space-time-frequency CF $R_{L}(t ; \Delta t, \Delta f, \Delta x)$, and 4D $\mathrm{LSF} C_{H}(t, \tau, \nu, \Omega)$, respectively. The time-delay-variant space cross-correlation function (CCF) $\rho(t, \tau ; \Delta x)$ and time-delayvariant autocorrelation function (ACF) $r(t, \tau ; \Delta t)$ can be obtained from $R_{h}(t, \tau, \Delta t, \Delta x)$ by imposing $\Delta t=0$ and $\Delta x=0$, respectively. We can also derive the time-delay variant $\mathrm{SD}$ PSD $W(t, \tau, \nu, \Delta x)$ by applying the Fourier transform to $R_{h}(t, \tau, \Delta t, \Delta x)$ in terms of $\Delta t$. The relationship between the CFs of non-stationary HST channel models is illustrated in Fig. 1, which serves as a fundamental framework for Sections III and IV. By applying the WSS assumption, the CFs will not depend on $t$ and the 4D CFs will be further reduced to 3D ones as in [36]. Finally, the spatial extension introduced in this section should not be confused with the one presented in [40] where the spatial dimension is related directly to the receiver position.

\section{THE NON-STATIONARY WIDEBAND THEORETICAL GBSM}

\section{A. Description of the Wideband MIMO Theoretical Model}

We adopt the IMT-A cellular network architecture for the HST communication system where MRSs are deployed on the surface of the train. Therefore, the end-to-end communications between the BS and MS will consist of two channels: outdoor channel and indoor one as illustrated in Fig. 2. Here, we 


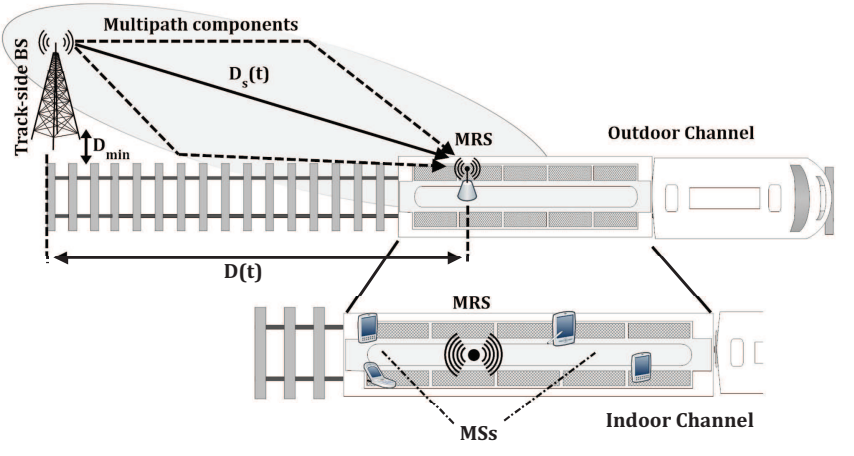

Fig. 2. A HST communication system deploying MRSs.

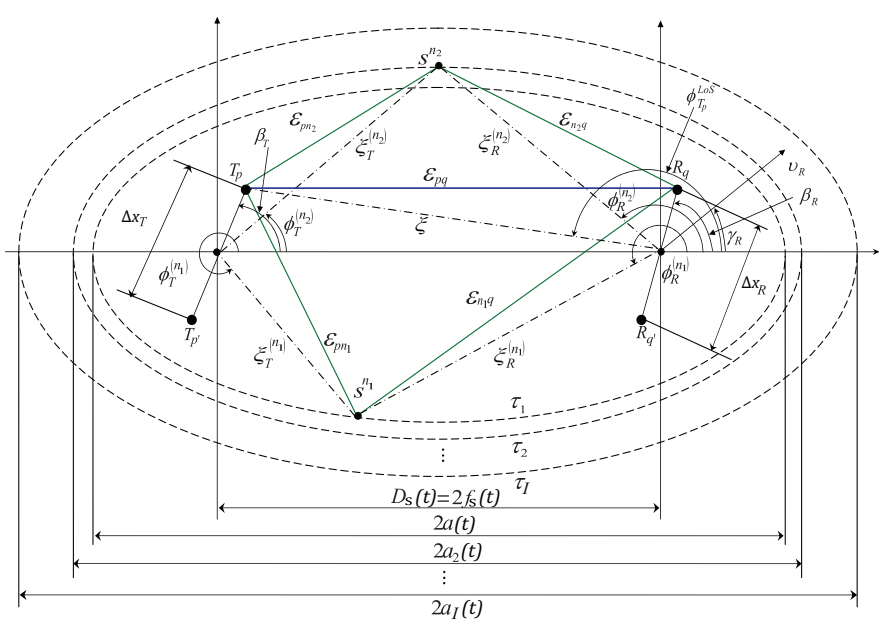

Fig. 3. The GBSM for a wideband MIMO HST channel.

will focus on the outdoor channel between the BS and MRS in RMa scenarios. We consider a MIMO HST system with $S$ transmit and $U$ receive omni-directional antenna elements. The BS is considered to be located on the track-side with the minimum distance between the BS and the track denoted as $D_{\min }=50 \mathrm{~m}$ [11]. The time-varying distance between the BS and MRS is $D_{s}(t)=\sqrt{\left(D_{\min }^{2}+D^{2}(t)\right)}$, where $D(t)$ stands for the projection of $D_{s}(t)$ on the railway track plane. Fig. 3 illustrates the proposed GBSM, which consists of multiple confocal ellipses with single-bounced rays and the line-of-sight (LoS) component [?]. Geometric elliptical channel models have widely been used to model wideband MIMO channels [41]-[43]. Other GBSMs like one-ring and two-ring models have been mainly used to model narrowband MIMO channels and their extension to wideband channel models [44], [45] is not straightforward. For clarity purposes, we use a $2 \times 2$ MIMO channel model in Fig. 3 as an example. The parameters in Fig. 3 are defined in Table I.

Based on the tapped delay line (TDL) structure, the taps are represented by multiple confocal ellipses with the BS and MRS located at the foci. There are $N_{i}$ effective scatterers on the $i$ th ellipse (i.e., $i$ th tap), where $i=1,2, \ldots, I$ and $I$ is the total number of ellipses or taps. Each effective scatterer is intended to represent the effect of many physical scatterers within the region. The semi-major axis of the $i$ th ellipse and the $n_{i}$ th $\left(n_{i}=1, \ldots, N_{i}\right)$ effective scatterer are denoted by $a_{i}(t)$ and $s^{\left(n_{i}\right)}$, respectively. We denote the time-varying semi-minor axis of the $i$ th ellipse as $b_{i}(t)=\sqrt{a_{i}^{2}(t)-f_{s}^{2}(t)}$, where $f_{s}(t)=D_{s}(t) / 2$ represents a half of the distance between the two foci of ellipses. The tilt angles of the BS and MRS antenna arrays are denoted by $\beta_{T}$ and $\beta_{R}$, respectively. The MRS moves with the same speed $v_{R}$ as the train in the direction determined by the angle of motion $\gamma_{R}$. The AoA of the wave traveling from an effective scatterer $s^{\left(n_{i}\right)}$ to the MRS is denoted by $\phi_{R}^{\left(n_{i}\right)}(t)$. The AoD of the wave that impinges on the effective scatterer $s^{\left(n_{i}\right)}$ is denoted by $\phi_{T}^{\left(n_{i}\right)}(t)$, while $\phi_{T_{p}}^{\mathrm{LoS}}(t)$ denotes the AoA of a LoS path.

Based on the TDL concept, the complex space-time-variant channel impulse response between the $p$ th $(p=1, \ldots, S)$ element of the BS, $T_{p}$, and the $q$ th $(q=1, \ldots, U)$ element of the MRS, $R_{q}$, can be expressed as $h(t, \tau, x)=h_{p q}(t, \tau)=$ $\sum_{i=1}^{I} h_{i, p q}(t) \delta\left(\tau-\tau_{i}\right)$, where $h_{i, p q}(t)$ and $\tau_{i}$ denote the complex space-time-variant tap coefficients and the discrete propagation delay of the $i$ th tap, respectively. Note that the space domain $x$ is implicitly expressed by the subscript $p q$. From the above GBSM, the complex tap coefficients for the first tap ( $i=1)$ of the $T_{p}-R_{q}$ link is a superposition of the LoS component and single-bounced (SB) components, and can be expressed as $h_{p q}(t, \tau)$ and $h_{i, p q}(t)$ depend only on the antenna spacings of the BS and MRS denoted by $\Delta x_{T}$ and $\Delta x_{R}$, respectively.

$$
h_{1, p q}(t)=h_{1, p q}^{\mathrm{LoS}}(t)+h_{1, p q}^{\mathrm{SB}}(t)
$$

where

$$
h_{1, p q}^{\mathrm{LoS}}(t)=\sqrt{\frac{K_{p q}}{K_{p q}+1}} e^{-j 2 \pi f_{c} \tau_{p q}(t)} e^{j 2 \pi f_{\max } t \cos \left(\phi_{T_{p}}^{\operatorname{LoS}}(t)-\gamma_{R}\right)}
$$

$$
\begin{gathered}
h_{1, p q}^{\mathrm{SB}}(t)=\sqrt{\frac{\Omega_{1, p q}}{K_{p q}+1}} \lim _{N_{1} \rightarrow \infty} \sum_{n_{1}=1}^{N_{1}} \frac{1}{\sqrt{N_{1}}} e^{j\left(\psi_{n_{1}}-2 \pi f_{c} \tau_{p q, n_{1}}(t)\right)} \\
\times e^{j 2 \pi f_{\max } t \cos \left(\phi_{R}^{\left(n_{1}\right)}(t)-\gamma_{R}\right)} .
\end{gathered}
$$

The complex tap coefficients for other taps $(1<i \leq I)$ of the $T_{p}-R_{q}$ link is a sum of $\mathrm{SB}$ components only and can be expressed as

$$
\begin{aligned}
& h_{i, p q}(t)=h_{i, p q}^{\mathrm{SB}}(t)=\sqrt{\Omega_{i, p q}} \lim _{N_{i} \rightarrow \infty} \sum_{n_{i}=1}^{N_{i}} \frac{1}{\sqrt{N_{i}}} \\
& \times e^{j\left(\psi_{n_{i}}-2 \pi f_{c} \tau_{p q, n_{i}}(t)\right)} e^{j 2 \pi f_{\max } t \cos \left(\phi_{R}^{\left(n_{i}\right)}(t)-\gamma_{R}\right)}, 1<i \leq I .
\end{aligned}
$$

It is worth mentioning that in (7a), (7b), and (8), we have timevarying parameters $\tau_{p q}(t), \phi_{T_{p}}^{\mathrm{LoS}}(t), \tau_{p q, n_{i}}(t)(i=1, \ldots, I)$, and $\phi_{R}^{\left(n_{i}\right)}(t)$, which make the underlying GBSM a non-stationary one. If these parameters are not time-varying, then the GBSM can be reduced to a WSS one, as proposed in [?]. In the following, our task is to define or calculate the above timevarying parameters.

In (7a), (7b), and (8), $\Omega_{i, p q}$ designates the mean power for the $i$ th tap, $\tau_{p q}(t)=\varepsilon_{p q}(t) / c$, and $\tau_{p q, n_{i}}(t)=\left(\varepsilon_{p n_{i}}(t)+\right.$ 
TABLE I

DEFINITION OF PARAMETERS IN FIG. 3.

\begin{tabular}{|c|c|}
\hline Parameters & Definition \\
\hline$D_{s}(t)$ & distance between the BS and MRS \\
\hline$f_{s}(t)$ & half length of the distance between the two foci of ellipses \\
\hline$a_{i}(t), b_{i}(t)$ & semi-major axis and semi-minor axis of the $i$ th ellipse, respectively \\
\hline$v_{R}, \gamma_{R}$ & MRS speed and angle of motion, respectively \\
\hline$\Delta x_{T}, \Delta x_{R}$ & antenna element spacings of the BS and MRS, respectively \\
\hline$\beta_{T}, \beta_{R}$ & tilt angles of the BS and MRS antenna arrays in the $\mathrm{x}-\mathrm{y}$ plane (relative to the $\mathrm{x}$-axis), respectively \\
\hline$\phi_{T_{p}}^{\mathrm{LoS}}(t), \phi_{R}^{\left(n_{i}\right)}(t)$ & AoA of the LoS path and AoA of the wave travelling from an effective scatterer $s^{\left(n_{i}\right)}$ to the MRS, respectively \\
\hline$\phi_{T}^{\left(n_{i}\right)}(t)$ & AoD of the wave that impinges on the effective scatterer $s^{\left(n_{i}\right)}$ \\
\hline$\xi, \xi_{T}^{\left(n_{i}\right)}(t)$, and $\xi_{R}^{\left(n_{i}\right)}(t)$ & distances $d\left(T_{p}, \mathrm{MRS}\right), d\left(\mathrm{BS}, s^{\left(n_{i}\right)}\right)$, and $d\left(s^{\left(n_{i}\right)}, \mathrm{MRS}\right)$, respectively \\
\hline$\varepsilon_{p q}, \varepsilon_{p n_{i}}, \varepsilon_{n_{i} q}$ & distances $d\left(T_{p}, R_{q}\right), d\left(T_{p}, s^{\left(n_{i}\right)}\right)$, and $d\left(s^{\left(n_{i}\right)}, R_{q}\right)$, respectively \\
\hline
\end{tabular}

$\left.\varepsilon_{n_{i} q}(t)\right) / c$ are the travel times of the waves through the links $T_{p}-R_{q}$ and $T_{p}-s^{\left(n_{i}\right)}-R_{q}$, respectively, as shown in Fig. 3. Here, $c$ represents the speed of light and the symbol $K_{p q}$ designates the Ricean factor. The phases $\psi_{n_{1}}$ and $\psi_{n_{i}}$ are independent and identically distributed (i.i.d.) random variables with uniform distributions over $[-\pi, \pi)$ and $f_{\max }$ is the maximum Doppler shift related to the MRS. From Fig. 3 and based on the law of cosines, we have [43]

$$
\begin{aligned}
& \varepsilon_{p q}(t) \approx D_{s}(t)-k_{p} \Delta x_{T} \cos \beta_{T}-k_{q} \Delta x_{R} \cos \left(\phi_{T_{p}}^{\mathrm{LoS}}(t)-\beta_{R}\right) \\
& \varepsilon_{p n_{i}}(t) \approx \xi_{T}^{\left(n_{i}\right)}(t)-k_{p} \Delta x_{T} \cos \left(\phi_{T}^{\left(n_{i}\right)}(t)-\beta_{T}\right) \\
& \varepsilon_{n_{i} q}(t) \approx \xi_{R}^{\left(n_{i}\right)}(t)-k_{q} \Delta x_{R} \cos \left(\phi_{R}^{\left(n_{i}\right)}(t)-\beta_{R}\right)
\end{aligned}
$$

where $k_{p}=(S-2 p+1) / 2, \quad k_{q}=(U-2 q+1) / 2$, and $\xi_{R}^{\left(n_{i}\right)}(t)=b_{i}^{2}(t) /\left(a_{i}(t)+f_{s}(t) \cos \phi_{R}^{\left(n_{i}\right)}(t)\right)$ with $\xi_{T}^{\left(n_{i}\right)}(t)=$ $\left(a_{i}^{2}(t)+f_{s}^{2}(t)+2 a_{i}(t) f_{s}(t) \cos \phi_{R}^{\left(n_{i}\right)}(t)\right) /\left(a_{i}(t)+f_{s}(t) \cos \phi_{R}^{\left(n_{i}\right)}(t)\right)$

Note that the AoD $\phi_{T}^{\left(n_{i}\right)}(t)$ and AoA $\phi_{R}^{\left(n_{i}\right)}(t)$ are interdependent for SB rays. The relationship between the AoD and AoA for multiple confocal ellipses model can be given by [43]

$$
\begin{gathered}
\sin \phi_{T}^{\left(n_{i}\right)}(t)=\frac{b_{i}^{2}(t) \sin \phi_{R}^{\left(n_{i}\right)}(t)}{a_{i}^{2}(t)+f_{s}^{2}(t)+2 a_{i}(t) f_{s}(t) \cos \phi_{R}^{\left(n_{i}\right)}(t)} \\
\cos \phi_{T}^{\left(n_{i}\right)}(t)=\frac{2 a_{i}(t) f_{s}(t)+\left(a_{i}^{2}(t)+f_{s}^{2}(t)\right) \cos \phi_{R}^{\left(n_{i}\right)}(t)}{a_{i}^{2}(t)+f_{s}^{2}(t)+2 a_{i}(t) f_{s}(t) \cos \phi_{R}^{\left(n_{i}\right)}(t)}
\end{gathered}
$$

The time-variant LoS AoA $\phi_{T_{p}}^{\mathrm{LoS}}(t)$ can be expressed as [5]

$\phi_{T_{p}}^{\operatorname{LoS}}(t)=\left\{\begin{array}{l}\phi_{T_{p}}^{\operatorname{LoS}}\left(t_{0}\right)+\arccos \left(\frac{D_{s}\left(t_{0}\right)+v_{R} t \cos \gamma_{R}}{D_{s}(t)}\right),-\pi \leq \gamma_{R} \leq 0 \\ \phi_{T_{p}}^{\operatorname{LoS}}\left(t_{0}\right)-\arccos \left(\frac{D_{s}\left(t_{0}\right)+v_{R} t \cos \gamma_{R}}{D_{s}(t)}\right), 0 \leq \gamma_{R} \leq \pi\end{array}\right.$

where $\phi_{T_{p}}^{\operatorname{LoS}}\left(t_{0}\right)=\arcsin \left(\frac{k_{p} \Delta x_{T}}{D_{s}\left(t_{0}\right)} \sin \beta_{T}\right)$ denotes the initial LoS AoA at time $t=t_{0}$.

We assume that the numbers of effective scatterers in the theoretical model tend to infinity, i.e., $N_{i} \rightarrow \infty$. In this case, the discrete angles $\phi_{T}^{\left(n_{i}\right)}(t)$ and $\phi_{R}^{\left(n_{i}\right)}(t)$ can be replaced by continuous ones $\phi_{T}^{(i)}(t)$ and $\phi_{R}^{(i)}(t)$, respectively. scatterer distributions like the uniform and Gaussian PDFs. Here, we use the von Mises PDF to describe the time-varying angles

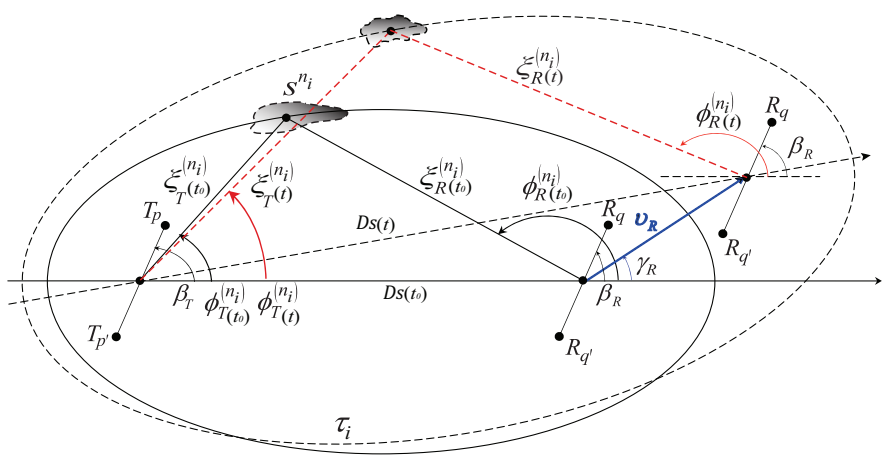

Fig. 4. The time-varying angular parameters in the HST channel model.

$\phi_{T}^{\left(n_{i}\right)}(t)$ and $\phi_{R}^{\left(n_{i}\right)}(t)$ as it is a general function and includes some well-known PDFs as special cases, e.g., uniform and Gaussian PDFs [43]. The von Mises PDF is defined as $f(\phi) \triangleq \exp [k \cos (\phi-\mu)] /\left[2 \pi I_{0}(k)\right]$, where $\mu$ is the mean value of angle $\phi \in[-\pi, \pi), I_{0}(\cdot)$ is the zeroth-order modified Bessel function of the first kind, and $k(k \geq 0)$ is a positive realvalued parameter that controls the spread of $\phi$. Applying the von Mises distribution to the time-varying AoAs, we get $f\left(\phi_{R}^{(i)}\right)(t) \triangleq \exp \left[k_{R}^{(i)} \cos \left(\phi_{R}^{(i)}-\mu_{R}^{(i)}(t)\right)\right] /\left[2 \pi I_{0}\left(k_{R}^{(i)}\right)\right]$, where $\mu_{R}^{(i)}$ is the mean angular value of the AoA $\phi_{R}^{(i)}$ and $k_{R}^{(i)}$ is the relevant von Mises parameter that controls the spread of $\phi_{R}^{(i)}$. Similarly, we can get $f\left(\phi_{T}^{(i)}\right)(t)$ with $\mu_{T}^{(i)}$ and $k_{T}^{(i)}$.

In Fig. 4, the MRS is moving with the speed of $v_{R}$ in the direction defined by the angle of motion $\gamma_{R}$. Correspondingly, the AoAs and the axes of the ellipses will be changed. The distance between the BS and the MRS can be calculated as $D_{s}(t)=2 f_{s}(t)=$ $\left(\sqrt{D_{s}^{2}\left(t_{0}\right)+\left(v_{R} t\right)^{2}+2 D_{s}\left(t_{0}\right) v_{R} t \cos \gamma_{R}}\right)$. Based on the geometric relations and by defining all the angles in Fig. 4, the time-varying function of mean AoA $\mu_{R}^{(i)}(t)$ can be derived as (12) [46], which is presented at the top of the next page.

\section{B. Statistical Properties of the Theoretical Model}

In this subsection, we will derive the statistical properties of the proposed non-stationary HST GBSM based on the theoretical framework described in Section II under the US and antenna stationarity assumptions. 


$$
\mu_{R}^{(i)}(t)= \begin{cases}\gamma_{R}-\arccos \left(\frac{v_{R} t-\xi_{R}^{\left(n_{i}\right)}\left(t_{0}\right) \cos \left(\gamma_{R}-\mu_{R}^{(i)}\left(t_{0}\right)\right)}{\sqrt{\xi_{R}^{2\left(n_{i}\right)}\left(t_{0}\right)+\left(v_{R} t\right)^{2}-2 \xi_{R}^{\left(n_{i}\right)}\left(t_{0}\right) v_{R} t \cos \left(\gamma_{R}-\mu_{R}^{i}\left(t_{0}\right)\right)}}\right), & -\pi \leq \gamma_{R} \leq 0 \\ \gamma_{R}+\arccos \left(\frac{v_{R} t-\xi_{R}^{\left(n_{i}\right)}\left(t_{0}\right) \cos \left(\gamma_{R}-\mu_{R}^{(i)}\left(t_{0}\right)\right)}{\sqrt{\xi_{R}^{2\left(n_{i}\right)}\left(t_{0}\right)+\left(v_{R} t\right)^{2}-2 \xi_{R}^{\left(n_{i}\right)}\left(t_{0}\right) v_{R} t \cos \left(\gamma_{R}-\mu_{R}^{i}\left(t_{0}\right)\right)}}\right), & 0 \leq \gamma_{R} \leq \pi .\end{cases}
$$

1) Time-variant $S T C F$ : The correlation properties of two arbitrary channel impulse responses $h_{p q}(t, \tau)$ and $h_{p^{\prime} q^{\prime}}(t, \tau)$ of a wideband MIMO HST channel are determined by the correlation properties of $h_{i, p q}(t)$ and $h_{i, p^{\prime} q^{\prime}}(t)$ in each tap, since there is no correlation between the underlying processes in different taps. The normalized time-variant ST CF can be derived as

$$
R_{h}\left(t, \Delta x_{T}, \Delta x_{R}, \Delta t\right)=\frac{\mathrm{E}\left\{h_{i, p q}(t) h_{i, p^{\prime} q^{\prime}}^{*}(t-\Delta t)\right\}}{\sqrt{\Omega_{i, p q} \Omega_{i, p^{\prime} q^{\prime}}}}
$$

which can be obtained from the time-delay variant space CCF $R_{h}(t, \tau ; \Delta t, \Delta x)$ in Fig. 1 with $\tau=0$.

- In the case of the LoS component,

$$
\begin{aligned}
R_{h}^{\mathrm{LoS}}\left(t, \Delta x_{T}, \Delta x_{R}, \Delta t\right) & =K^{\prime} e^{j 2 \pi\left[P \cos \beta_{T}-Q \cos \left(\phi_{T_{p}}^{\mathrm{LoS}}(t)-\beta_{R}\right)\right]} \\
& \times e^{j 2 \pi f_{\max } \cos \left(\phi_{T_{p}}^{\mathrm{LoS}}(t-\Delta t)-\gamma_{R}\right) \Delta t}
\end{aligned}
$$

where $P=\left(p^{\prime}-p\right) \Delta x_{T} / \lambda, Q=\left(q^{\prime}-q\right) \Delta x_{R} / \lambda$, and $K^{\prime}=$

$$
\sqrt{\frac{K_{p q} K_{p^{\prime} q^{\prime}}}{\left(K_{p q}+1\right)\left(K_{p^{\prime} q^{\prime}}+1\right)}} \text {. }
$$

- In the case of the SB component,

$$
\begin{aligned}
& R_{h}^{\mathrm{SB}}\left(t, \Delta x_{T}, \Delta x_{R}, \Delta t\right)=\frac{1}{2 \pi I_{0}\left(k_{R}^{(i)}\right) U} \\
& \times \int_{-\pi}^{\pi} e^{k_{R}^{(i)} \cos \left(\phi_{R}^{(i)}-\mu_{R}^{(i)}(t)\right)} e^{j 2 \pi\left[P \cos \left(\phi_{T}^{(i)}-\beta_{T}\right)+Q \cos \left(\phi_{R}^{(i)}-\beta_{R}\right)\right]} \\
& \quad \times e^{j 2 \pi \xi_{T R}^{\left(n_{i}\right)}(t, \Delta t)} e^{j 2 \pi f_{\max } \cos \left(\phi_{R}^{(i)}-\gamma_{R}\right) \Delta t} \mathrm{~d} \phi_{R}^{(i)}
\end{aligned}
$$

where $\xi_{T R}^{n_{i}}(t, \Delta t)=\xi_{T}^{n_{i}}(t-\Delta t)-\xi_{T}^{n_{i}}(t)+\xi_{R}^{n_{i}}(t-\Delta t)-$ $\xi_{R}^{n_{i}}(t)$.

By imposing $\Delta t=0$ in (13), we get the normalized timevariant space CCF between two arbitrary channel coefficients as

$$
\begin{aligned}
\rho\left(t, \Delta x_{T}, \Delta x_{R}\right)=\frac{\mathrm{E}\left\{h_{i, p q}(t) h_{i, p^{\prime} q^{\prime}}^{*}(t)\right\}}{\sqrt{\Omega_{i, p q} \Omega_{i, p^{\prime} q^{\prime}}}} \\
\quad=R_{h}\left(t, \Delta x_{T}, \Delta x_{R}, 0\right) .
\end{aligned}
$$

- In the case of the LoS component,

$\rho^{\mathrm{LoS}}\left(t, \Delta x_{T}, \Delta x_{R}\right)=K^{\prime} e^{j 2 \pi\left[P \cos \beta_{T}-Q \cos \left(\phi_{T_{p}}^{\mathrm{LOS}}(t)-\beta_{R}\right)\right]}$.

- In the case of the SB component,

$$
\begin{gathered}
\rho^{\mathrm{SB}_{i}}\left(t, \Delta x_{T}, \Delta x_{R}\right)=\frac{1}{2 \pi I_{0}\left(k_{R}^{(i)}\right) U} \int_{-\pi}^{\pi} e^{k_{R}^{(i)} \cos \left(\phi_{R}^{(i)}-\mu_{R}^{(i)}(t)\right)} \\
\times e^{j 2 \pi\left[P \cos \left(\phi_{T}^{(i)}-\beta_{T}\right)+Q \cos \left(\phi_{R}^{(i)}-\beta_{R}\right)\right]} \mathrm{d} \phi_{R}^{(i)} .
\end{gathered}
$$

The normalized time-variant space CCF for the first tap $(i=1)$ can be expressed as the summation of (17) and (18) with $i=1$, i.e.,

$$
\rho_{1}\left(t, \Delta x_{T}, \Delta x_{R}\right)=\rho^{\mathrm{LoS}}\left(t, \Delta x_{T}, \Delta x_{R}\right)+\rho^{\mathrm{SB}_{1}}\left(t, \Delta x_{T}, \Delta x_{R}\right) .
$$

Similarly, the normalized time-variant ACF can be obtained by imposing $\Delta x_{T}=0$ and $\Delta x_{R}=0$ in (13), i.e.,

$$
r(t, \Delta t)=\frac{\mathrm{E}\left\{h_{i, p q}(t) h_{i, p q}^{*}(t-\Delta t)\right\}}{\sqrt{\Omega_{i, p q} \Omega_{i, p^{\prime} q^{\prime}}}}=R_{h}(t, 0,0, \Delta t) .
$$

- In the case of the LoS component,

$$
r^{\mathrm{LoS}}(t, \Delta t)=\frac{K_{p q}}{1+K_{p q}} e^{j 2 \pi f_{\max } \cos \left(\phi^{\mathrm{LoS}}(t-\Delta t)-\gamma_{R}\right) \Delta t} .
$$

- In the case of the SB component,

$$
\begin{gathered}
r^{\mathrm{SB}_{i}}(t, \Delta t)=\frac{1}{2 \pi I_{0}\left(k_{R}^{(i)}\right)\left(1+K_{p q}\right)} \int_{-\pi}^{\pi} e^{k_{R}^{(i)} \cos \left(\phi_{R}^{(i)}-\mu_{R}^{(i)}(t)\right)} \\
\times e^{j 2 \pi \xi_{T R}^{\left(n_{i}\right)}(t, \Delta t)} e^{j 2 \pi f_{\max } \cos \left(\phi_{R}^{(i)}-\gamma_{R}\right) \Delta t} \mathrm{~d} \phi_{R}^{(i)}
\end{gathered}
$$

Therefore, the normalized time-variant ACF for the first tap $(i=1)$ can be expressed as the summation of (21) and (22) with $i=1$, i.e.,

$$
r_{1}(t, \Delta t)=r^{\mathrm{LoS}}(t, \Delta t)+r^{\mathrm{SB}_{1}}(t, \Delta t) .
$$

2) Time-variant SD PSD: The time-variant SD PSD can be obtained from the time-variant ST CF by applying the Fourier transformation in terms of $\Delta t$, i.e.,

$$
W\left(t, \nu, \Delta x_{T}, \Delta x_{R}\right)=\int R_{h}\left(t, \Delta x_{T}, \Delta x_{R}, \Delta t\right) e^{-j 2 \pi \nu \Delta t} \mathrm{~d} \Delta t .
$$

- In case of the LoS component,

$W^{\operatorname{LoS}}\left(t, \nu, \Delta x_{T}, \Delta x_{R}\right)=K^{\prime} e^{j 2 \pi\left[P \cos \beta_{T}-Q \cos \left(\phi_{T_{p}}^{\operatorname{LoS}}(t)-\beta_{R}\right)\right]}$

$$
\times \delta\left(\nu-\left(G(t)+v_{R}\right)\right)
$$

where $G(t)=f_{\max } \cos \left(\phi_{T_{p}}^{\mathrm{LoS}}(t)-\gamma_{R}\right)$.

- In case of the SB component,

$$
\begin{aligned}
W^{\mathrm{SB}_{i}}\left(t, \nu, \Delta x_{T}, \Delta x_{R}\right)=\int R_{h}^{\mathrm{SB}_{i}}( & \left.t, \Delta x_{T}, \Delta x_{R}, \Delta t\right) \\
& \times e^{-j 2 \pi \nu \Delta t} \mathrm{~d} \Delta t .
\end{aligned}
$$

This integral will have to be evaluated numerically. 
3) $L S F$ : It can be obtained from the time-variant spacetime-frequency CF $R_{L}(t ; \Delta t, \Delta f, \Delta x)$ using Fourier transform with respect to $\Delta t$ and $\Delta x$ and inverse Fourier transform with respect to $\Delta f$, i.e.,

$$
\begin{aligned}
C_{H}(t, \tau, \nu, \Omega)= & \iiint R_{L}(t ; \Delta t, \Delta f, \Delta x) \\
& \times e^{-j 2 \pi(\nu \Delta t-\tau \Delta f+\Omega \Delta x)} \mathrm{d} \Delta t \mathrm{~d} \Delta f \mathrm{~d} \Delta x .
\end{aligned}
$$

where the time-variant space-time-frequency $\mathrm{CF}$ can be obtained from (3) after considering the US and antenna stationarity assumptions, i.e.,

$R_{L}(t ; \Delta t, \Delta f, \Delta x)=\frac{\mathrm{E}\left\{L_{H_{p q}}(t, f) L_{H_{p^{\prime} q^{\prime}}}^{*}(t-\Delta t, f-\Delta f)\right\}}{\sqrt{\Omega_{i, p q} \Omega_{i, p^{\prime} q^{\prime}}}}$.

Here, $L_{H_{p q}}(t, f)$ is the space-time-variant transfer function that can be obtained by (1), i.e.,

$$
L_{H_{p q}}(t, f)=\int h_{p q}(t, \tau) e^{-j 2 \pi f \tau} \mathrm{d} \tau \text {. }
$$

- In the case of the LoS component,

$$
\begin{aligned}
& R_{L}^{\mathrm{LoS}}(t ; \Delta t, \Delta f, \Delta x)=K^{\prime} e^{j 2 \pi\left[P \cos \beta_{T}-Q \cos \left(\phi_{T_{p}}^{\mathrm{LoS}}(t)-\beta_{R}\right)\right]} \\
& \times e^{j 2 \pi f_{\max } \cos \left(\phi_{T_{p}}^{\mathrm{LoS}}(t)-\gamma_{R}\right) \Delta t-\Delta f \tau_{1}}
\end{aligned}
$$

where

$$
\begin{aligned}
C_{H}^{\mathrm{LoS}}(t, \tau, \nu, \Omega) & =\iiint R_{L}^{\mathrm{LoS}}(t ; \Delta t, \Delta f, \Delta x) \\
& \times e^{-j 2 \pi(\nu \Delta t-\tau \Delta f+\Omega \Delta x)} \mathrm{d} \Delta t \mathrm{~d} \Delta f \mathrm{~d} \Delta x .
\end{aligned}
$$

- In the case of the SB component,

$$
\begin{aligned}
C_{H}^{\mathrm{SB}_{i}}(t, \tau, \nu, \Omega) & =\iiint R_{L}^{\mathrm{SB}_{i}}(t ; \Delta t, \Delta f, \Delta x) \\
& \times e^{-j 2 \pi(\nu \Delta t-\tau \Delta f+\Omega \Delta x)} \mathrm{d} \Delta t \mathrm{~d} \Delta f \mathrm{~d} \Delta x
\end{aligned}
$$

where the time-variant space-time-frequency $\mathrm{CF}$ $R_{L}^{\mathrm{SB}_{i}}(t ; \Delta t, \Delta f, \Delta x)$ can be expressed as (33), which is shown at the top of the next page.

It is important to mention that all the investigated statistical properties in this section, i.e., $R_{h}\left(t, \Delta x_{T}, \Delta x_{R}, \Delta t\right)$, $\rho\left(t, \Delta x_{T}, \Delta x_{R}\right), \quad r(t, \Delta t), \quad W\left(t, \nu, \Delta x_{T}, \Delta x_{R}\right), \quad$ and $C_{H}(t, \tau, \nu, \Omega)$, are time-variant due to the non-stationarity of the proposed GBSM. For stationary channel models, the corresponding statistical properties are not dependent on $t$.

\section{The Simulation Model for Wideband MiMo HST CHANNELS}

\section{A. Description of the Wideband MIMO Simulation Model}

The proposed theoretical model assumes an infinite number of effective scatters and hence it cannot be used for simulations. Therefore, we need to develop a HST simulation model, which can be obtained from the theoretical one by utilizing only a finite number of scatterers $N$. The complex space-timevariant tap coefficient of the first tap of the link $T_{p}-R_{q}$ for the simulation model can be expressed as

$$
\tilde{h}_{1, p q}(t)=\tilde{h}_{1, p q}^{\mathrm{LoS}}(t)+\tilde{h}_{1, p q}^{\mathrm{SB}}(t)
$$

where

$\tilde{h}_{1, p q}^{\mathrm{LoS}}(t)=\sqrt{\frac{K_{p q}}{K_{p q}+1}} e^{-j 2 \pi f_{c} \tau_{p q}(t)} e^{j\left[2 \pi f_{\max } t \cos \left(\tilde{\phi}_{T_{p}}^{\mathrm{LoS}}(t)-\gamma_{R}\right)\right]}$

and

$$
\begin{array}{r}
\tilde{h}_{1, p q}^{\mathrm{SB}}(t)=\sqrt{\frac{\Omega_{1, p q}}{K_{p q}+1}} \sum_{n_{1}=1}^{N_{1}} \frac{1}{\sqrt{N_{1}}} e^{j\left(\psi_{n_{1}}-2 \pi f_{c} \tau_{p q, n_{1}}(t)\right)} \\
\times e^{j\left[2 \pi f_{\max } t \cos \left(\tilde{\phi}_{R}^{\left(n_{1}\right)}(t)-\gamma_{R}\right)\right] .}
\end{array}
$$

The complex space-time-variant channel coefficient for the rest of the taps $(1<i \leq I)$ can be expressed as

$$
\begin{aligned}
\tilde{h}_{i, p q}(t)= & \tilde{h}_{i, p q}^{\mathrm{SB}}(t)=\sqrt{\Omega_{i, p q}} \sum_{n_{i}=1}^{N_{i}} \frac{1}{\sqrt{N_{i}}} e^{j\left(\psi_{n_{i}}-2 \pi f_{c} \tau_{p q, n_{i}}(t)\right)} \\
& \times e^{j\left[2 \pi f_{\max } t \cos \left(\tilde{\phi}_{R}^{\left(n_{i}\right)}(t)-\gamma_{R}\right)\right]}, 1<i \leq I
\end{aligned}
$$

By comparing the simulation and theoretical models and considering the fact that the AoDs are related to the AoAs, we only need to determine the discrete AoA $\left\{\tilde{\phi}_{R}^{\left(n_{i}\right)}\right\}_{n_{i}=1}^{N_{i}}$ for the simulation model. In case of isotropic scattering, i.e., $k_{R}^{(i)}=0$, the quantities $\left\{\tilde{\phi}_{R}^{\left(n_{i}\right)}\right\}_{n_{i}=1}^{N_{i}}$ should preferably be computed using the extended method of exact Doppler spread (EMEDS) [42], while MMEA and the $L p$-norm method (LPNM) provide high quality solutions when the scattering is non-isotropic [42]. Here, we consider a non-isotropic scattering environment and hence we will use MMEA to calculate $\left\{\tilde{\phi}_{R}^{\left(n_{i}\right)}\right\}_{n_{i}=1}^{N_{i}}$ for the simulation model. By applying the MMEA, the AoAs $\left\{\tilde{\phi}_{R}^{\left(n_{i}\right)}\right\}_{n_{i}=1}^{N_{i}}$ can be determined by finding the solutions of the following equation using numerical root-finding techniques [42]:

$$
\frac{n_{i}-\frac{1}{4}}{N_{i}}-\int_{\mu_{R}^{(i)}\left(t_{0}\right)-\pi}^{\tilde{\phi}_{R}^{\left(n_{i}\right)}} f\left(\phi_{R}^{(i)}\right)\left(t_{0}\right) \mathrm{d} \phi_{R}^{(i)}=0, \quad n_{i}=1,2, \ldots, N_{i} .
$$

Reasonable values of $N_{i}$ are in the range from 40 to 50 [42].

\section{B. Statistical Properties of the Simulation Model}

Based on our wideband MIMO HST theoretical model and its statistical properties, the corresponding statistical properties for the simulation model can be derived by using discrete angle parameters.

1) Time-variant $S T C F$ : The normalized time-variant $\mathrm{ST}$ $\mathrm{CF}$ can be calculated by

$$
\tilde{R}_{h}\left(t, \Delta x_{T}, \Delta x_{R}, \Delta t\right)=\frac{\mathrm{E}\left\{\tilde{h}_{i, p q}(t) \tilde{h}_{i, p^{\prime} q^{\prime}}^{*}(t-\Delta t)\right\}}{\sqrt{\Omega_{i, p q} \Omega_{i, p^{\prime} q^{\prime}}}} .
$$

- In the case of the LoS component,

$$
\begin{gathered}
\tilde{R}_{h}^{\mathrm{LoS}}\left(t, \Delta x_{T}, \Delta x_{R}, \Delta t\right)=K^{\prime} e^{j 2 \pi\left[P \cos \beta_{T}-Q \cos \left(\tilde{\phi}_{T_{p}}^{\operatorname{LoS}}(t)-\beta_{R}\right)\right]} \\
\times e^{j 2 \pi f_{\max } \cos \left(\tilde{\phi}^{L o S}(t-\Delta t)-\gamma_{R}\right) \Delta t}
\end{gathered}
$$




$$
\begin{aligned}
R_{L}^{\mathrm{SB} i}(t ; \Delta t, \Delta f, \Delta x)=\frac{1}{2 \pi I_{0}\left(k_{R}^{(i)}\right) U} & \int_{-\pi}^{\pi} e^{k_{R}^{(i)} \cos \left(\phi_{R}^{(i)}-\mu_{R}^{(i)}(t)\right)} e^{j 2 \pi f_{\max } \cos \left(\phi_{R}^{(i)}-\gamma_{R}\right) \Delta t} \\
& \times e^{j 2 \pi\left[P \cos \left(\phi_{T}^{(i)}-\beta_{T}\right)+Q \cos \left(\phi_{R}^{(i)}-\beta_{R}\right)\right]} e^{-j 2 \pi \Delta f \tau_{i}} \mathrm{~d} \phi_{R}^{(i)} .
\end{aligned}
$$

- In the case of the SB component,

$$
\begin{aligned}
& \tilde{R}_{h}^{\mathrm{SB}}(\left.t, \Delta x_{T}, \Delta x_{R}, \Delta t\right)=\frac{1}{N_{i} U} \\
& \quad \times \sum_{n_{i}=1}^{N_{i}} e^{j 2 \pi\left[P \cos \left(\tilde{\phi}_{T}^{\left(n_{i}\right)}(t)-\beta_{T}\right)+Q \cos \left(\tilde{\phi}_{R}^{\left(n_{i}\right)}(t)-\beta_{R}\right)\right]} \\
& \quad \times e^{j 2 \pi \xi_{T R}^{\left(n_{i}\right)}(t, \Delta t)} e^{j 2 \pi f_{\max } \cos \left(\tilde{\phi}_{R}^{\left(n_{i}\right)}(t)-\gamma_{R}\right) \Delta t} .
\end{aligned}
$$

Similar to the procedure applied to the theoretical model, the normalized time-variant space $\mathrm{CCF}$ and the time-variant $\mathrm{ACF}$ of the simulation model can be expressed as

$$
\begin{gathered}
\tilde{\rho}\left(t, \Delta x_{T}, \Delta x_{R}\right)=\frac{\mathrm{E}\left\{\tilde{h}_{i, p q}(t) \tilde{h}_{i, p^{\prime} q^{\prime}}^{*}(t)\right\}}{\sqrt{\Omega_{i, p q} \Omega_{i, p^{\prime} q^{\prime}}}}=\tilde{R}_{h}\left(t, \Delta x_{T}, \Delta x_{R}, 0\right) \\
\tilde{r}(t, \Delta t)=\frac{\mathrm{E}\left\{\tilde{h}_{i, p q}(t) \tilde{h}_{i, p q}^{*}(t-\Delta t)\right\}}{\sqrt{\Omega_{i, p q} \Omega_{i, p^{\prime} q^{\prime}}}}=\tilde{R}_{h}(t, 0,0, \Delta t)
\end{gathered}
$$

respectively.

2) Time-variant SD PSD: The time-variant SD PSD of the simulation model $\tilde{W}\left(t, \nu, \Delta x_{T}, \Delta x_{R}\right)$ can be obtained from the ST CF by applying the Fourier transform in terms of $\Delta t$. Therefore, it can be expressed similar to (24) by replacing $R_{h}\left(t, \Delta x_{T}, \Delta x_{R}, \Delta t\right)$ by $\tilde{R}_{h}\left(t, \Delta x_{T}, \Delta x_{R}, \Delta t\right)$.

- In case of the LoS component, $\tilde{W}^{\mathrm{LoS}}\left(t, \nu, \Delta x_{T}, \Delta x_{R}\right)$ can be obtained by replacing $\phi_{T_{p}}^{\mathrm{LoS}}$ by $\tilde{\phi}_{T_{p}}^{\mathrm{LOS}}$ in (25).

- In case of the SB component, time-variant SD PSD $\tilde{W}^{\mathrm{SB}_{i}}\left(t, \nu, \Delta x_{T}, \Delta x_{R}\right)$ can be obtained from (26) by substituting $R_{h}^{\mathrm{SB}_{i}}\left(t, \Delta x_{T}, \Delta x_{R}, \Delta t\right)$ by $\tilde{R}_{h}^{\mathrm{SB}_{i}}\left(t, \Delta x_{T}, \Delta x_{R}, \Delta t\right)$.

3) LSF: Analogous to the theoretical model, the LSF of the simulation model $\tilde{C}_{H}(t, \tau, \nu, \Omega)$ can be obtained from the time-frequency-space CF $\tilde{R}_{L}(t ; \Delta t, \Delta f, \Delta x)$. Therefore, the derived equations can be obtained from (27)-(32) by replacing the theoretical model functions and the continuous model parameters, e.g., $\phi_{R}^{(i)}$ with the corresponding simulation model functions and discrete model parameters, e.g., $\tilde{\phi}_{R}^{\left(n_{i}\right)}$.

\section{Results AND ANALYSis}

In this section, the statistical properties of the proposed theoretical model and simulation model are evaluated and analyzed. Then, the proposed simulation channel model is further validated by measurements. The parameters for our analysis are listed here or specified otherwise: the LoS Ricean factor $K_{p q}=K_{p^{\prime} q^{\prime}}=6$, taps delays for the first and second taps are $\tau_{1}=0$ and $\tau_{2}=35 \mathrm{~ns}$, respectively, as for the RMa scenario in [10], $v_{R}=360 \mathrm{~km} / \mathrm{h}, f_{c}=4 \mathrm{GHz}$, and thus $f_{\max }=1.34 \mathrm{KHz}, D_{s}\left(t_{0}\right)=1000 \mathrm{~m}, D_{\min }=50 \mathrm{~m}$ as in

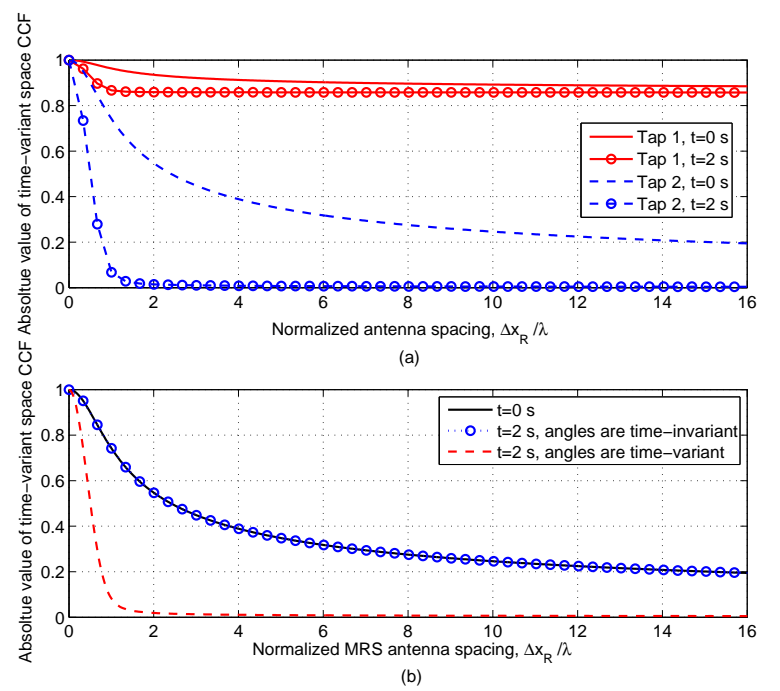

Fig. 5. (a) The absolute values of the time-variant space CCF of different taps of the proposed HST channel model at different time instants. (b) The absolute values of the time-variant space CCF of the second tap with/without time-varying angular parameters.

[5], $a_{1}\left(t_{0}\right)=\frac{D_{s}\left(t_{0}\right)}{2}+5 \mathrm{~m}, a_{2}\left(t_{0}\right)=\frac{D_{s}\left(t_{0}\right)}{2}+10.25 \mathrm{~m}$, $\xi_{R}^{\left(n_{i}\right)}\left(t_{0}\right)=600 \mathrm{~m}, \gamma_{R}=30^{\circ}, \mu_{R}^{(i)}\left(t_{0}\right)=45^{\circ}, k_{R}^{(i)}=6$ and we use a linear antenna array with $M_{R}=M_{T}=2$ and $\beta_{R}=\beta_{T}=60^{\circ}$.

\section{A. Time-variant ST CFs}

By adopting a BS antenna element spacing $\Delta x_{T}=\lambda$, the absolute values of the time-variant space CCF of non-stationary HST MIMO channel model are illustrated in Fig. 5(a) and Fig. 5(b). By using (19) and imposing $i=2$ in (18), Fig. 5(a) shows the absolute values of the time-variant space CCF of the first and second taps of the theoretical model at two different time instants, i.e., $t=0 \mathrm{~s}$ and $2 \mathrm{~s}$. From the figure, we can easily notice the higher correlation in the first tap in comparison with the second one because of the dominant LoS component. To highlight the impact of the time-varying parameters on channel statistical properties, Fig. 5(b) shows the absolute values of the time-variant space CCF of the second tap with time-variant and time-invariant angular parameters. It shows that time-varying angles are the only time-varying parameters that affect the time-variant space CCF. The same conclusion can be drawn from (18). Fig. 6 shows a comparison between the time-variant space CCFs of the theoretical model (18), simulation model (41), and simulation results with $N=50$ for BS antenna spacing $\Delta x_{T}=\lambda$ at different time instants $t=0 \mathrm{~s}$ and $2 \mathrm{~s}$. It shows that the simulation model provides a fairly good approximation 


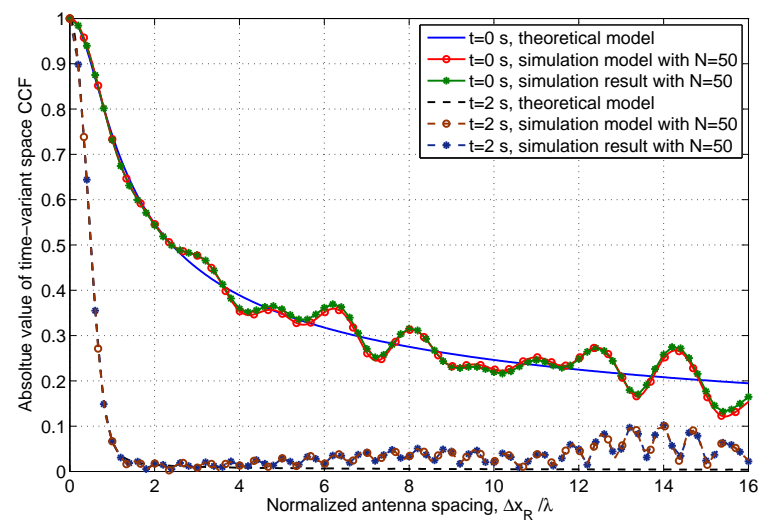

Fig. 6. Comparison between the time-variant space CCFs of the second tap of the theoretical model and simulation model for different time instants.
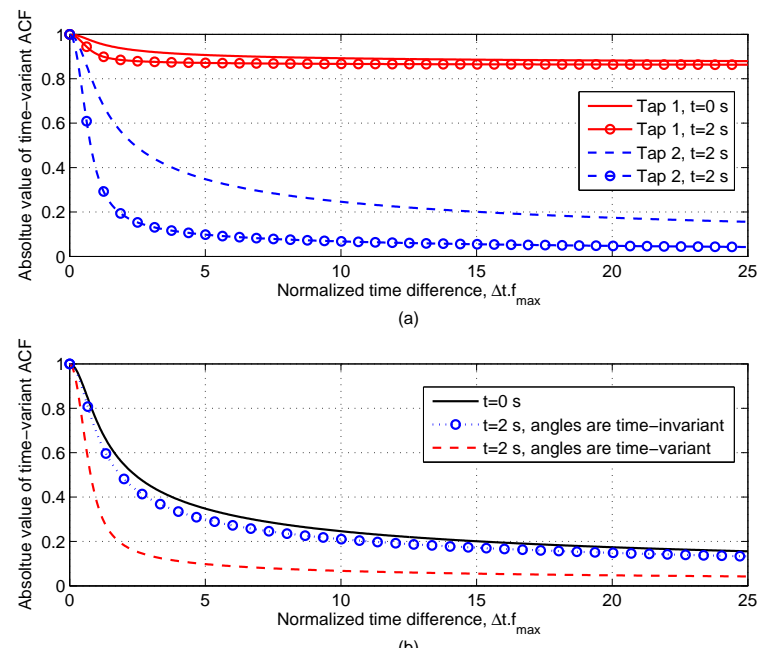

(b)

Fig. 7. (a) The absolute values of the time-variant ACF of different taps of the proposed HST channel model at different time instants. (b) The absolute values of the time-variant ACF with/without time-varying angular parameters.

to the theoretical one especially at small antenna spacings. The simulation results fit the simulation model very well, demonstrating the correctness of both theoretical derivations and simulations.

By using (23) and imposing $i=2$ in (18), Fig. 7(a) shows the absolute values of the time-variant ACF of different taps of the proposed theoretical HST channel model at different time instants. A higher correlation in the first tap in comparison with the second can be easily noticed. Again, this is due to the dominant LoS component. Fig. 7(b) shows the absolute values of the time-variant $\mathrm{ACF}$ of the second tap with/without time-varying angular parameters. We can see that even with time-invariant angles, the absolute value of the time-variant ACF still changes with time because of the time-varying dimensions of the ellipses. The same conclusion can be drawn from (22). From this figure, we can also calculate coherence time, which quantifies the duration within which the channel is approximately constant and can be defined as the smallest value of $\Delta t$ that fulfills the condition $|r(t, \Delta t)|=\frac{1}{2}|r(0, \Delta t)|$.

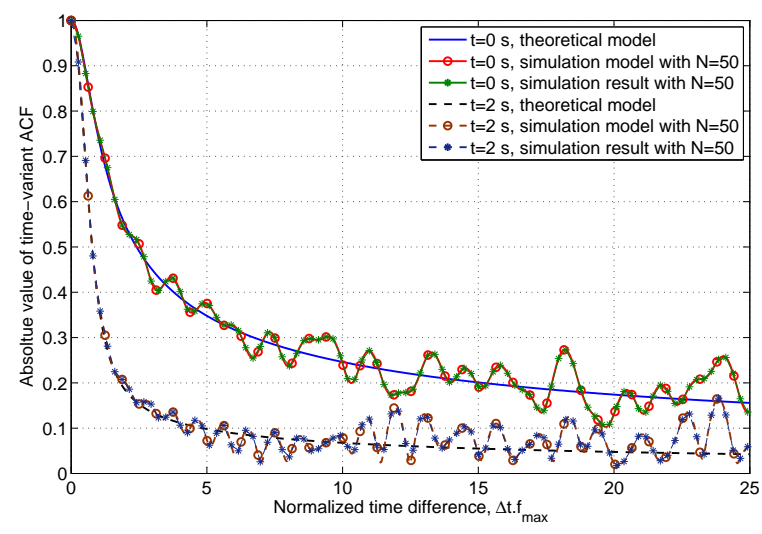

Fig. 8. Comparison between the time-variant ACFs of the second tap of the theoretical model and simulation model for different time instants.

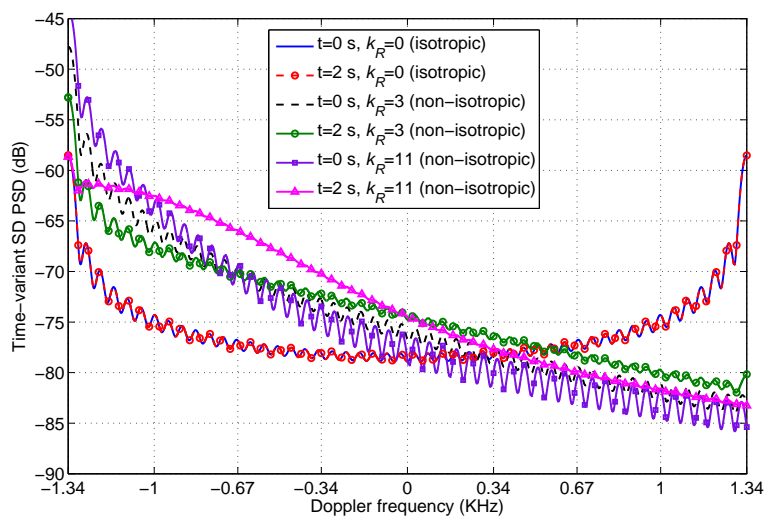

Fig. 9. Time-variant SD PSDs of the theoretical model for different scenarios $\left(v_{R}=360 \mathrm{~km} / \mathrm{h}, f_{\max }=1.34 \mathrm{KHz}\right)$ : isotropic: $k_{R}=0$ \& non-isotropic: $k_{R}=3$ $\& 11$.

The coherence time for time-variant and time-invariant angular parameters is equal to $1 \mathrm{~ms}$ and $4 \mathrm{~ms}$, respectively. It is important to mention that the smaller the coherence time, the larger the Doppler spread. Fig. 8 shows a comparison between the time-variant ACFs of the second tap of the theoretical model (22), simulation model (42), and simulation results for different time instants. Again, the simulation model provides a fairly good approximation to the theoretical one especially in small values of time separation. The simulation results and (42) of the simulation model match very well, illustrating the correctness of the derivation of (42) and simulations.

\section{B. Time-variant SD PSDs}

Fig. 9 compares the time-variant SD PSDs of the theoretical model (24) for isotropic (i.e., $k_{R}=0$ ) and non-isotropic (i.e., $\left.k_{R}>0\right)$ scenarios at different time instants with $\Delta x_{T}=$ $\Delta x_{R}=\lambda$. We can easily notice that the SD PSD is Ushaped for isotropic case only. To understand the impact of the angular parameters on SD PSD given in (26) for the theoretical model and the corresponding simulation model, Fig. 10 shows normalized SD PSDs of both models for different angular parameters, i.e., the angle of motion $\gamma_{R}$ and the initial mean 


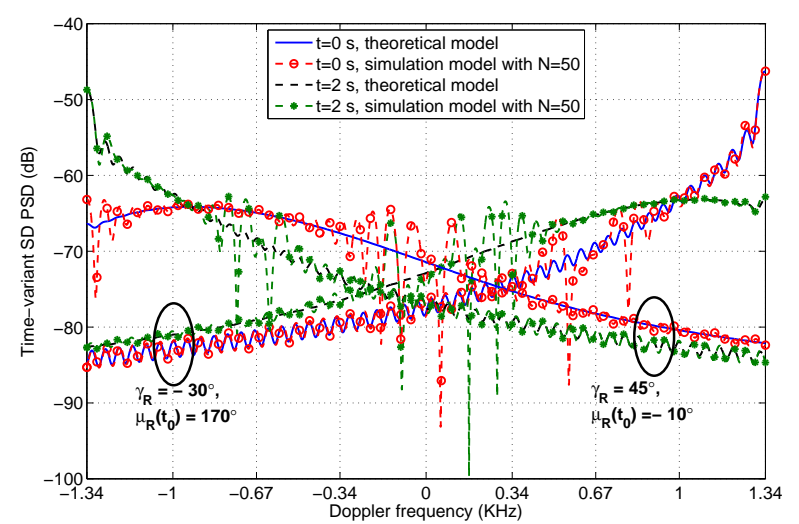

Fig. 10. Time-variant SD PSDs of the theoretical and simulation models for different angular parameters $\left(v_{R}=360 \mathrm{~km} / \mathrm{h}, f_{\max }=1.34 \mathrm{KHz}, k_{R}=11\right)$.

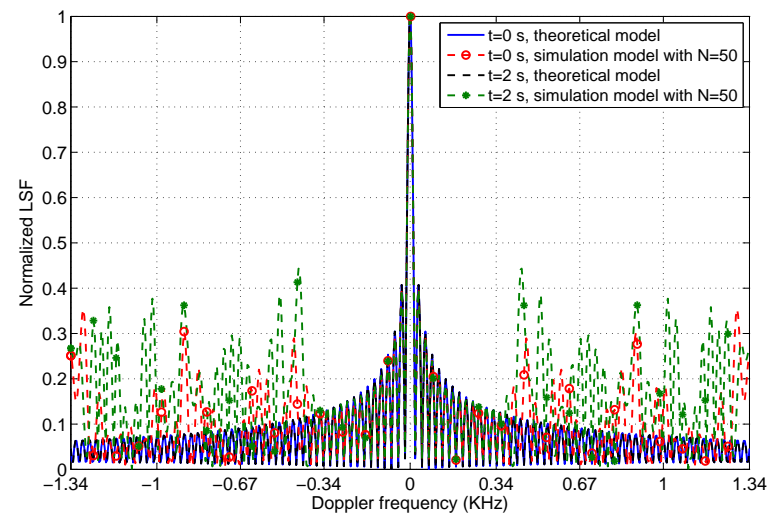

Fig. 11. Comparison between the LSFs of the second tap of the theoretical model and the simulation model for different time instants.

AoA $\mu_{R}\left(t_{0}\right)$. From this figure, it can be concluded that angular parameters of channel models affect considerably the trends of the time-variant SD PSDs.

\section{C. $L S F S$}

Fig. 11 shows a comparison between the LSFs of the theoretical model and simulation model with $N=50$ for different time instants. It can be noticed how the power of the effective scatterers varies with time $t$ and the power of the components with zero Doppler frequency is higher than the rest of the components. Simulation model shows good approximation to the theoretical one at different time instants. different time instants due to the non-stationarity of the HST channel. They also demonstrate that simulation model provides a good approximation to the statistical properties of the theoretical one.

\section{Stationary Interval}

To verify our proposed channel models, we use HST measurement data presented in [27] where the authors compared the stationary interval of a measured HST channel with that of standard channel models such as IMT-A and WINNER II.

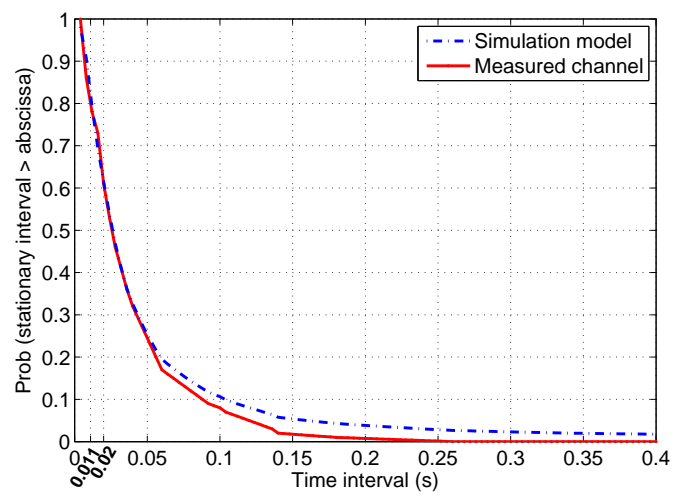

Fig. 12. The empirical CCDFs of stationary intervals for the proposed HST simulation model and the measured channel in [27].

The stationary interval can be calculated using averaged power delay profiles (APDPs) that can be expressed as [27]

$$
\overline{P_{h}}\left(t_{k}, \tau\right)=\frac{1}{N_{P D P}} \sum_{k}^{k+N_{P D P}-1}\left|\tilde{h}_{p q}\left(t_{k}, \tau\right)\right|^{2}
$$

where $N_{P D P}$ is the number of power delay profiles to be averaged, $t_{k}$ is the time of the $k$-th drop (snapshot), and $\tilde{h}_{p q}\left(t_{k}, \tau\right)=\sum_{i=1}^{I} \tilde{h}_{i, p q}\left(t_{k}\right) \delta\left(\tau-\tau_{i}\right)$. The correlation coefficient between two APDPs can be calculated as

$$
c\left(t_{k}, \Delta t\right)=\frac{\int \overline{P_{h}}\left(t_{k}, \tau\right) \overline{P_{h}}\left(t_{k}+\Delta t, \tau\right) \mathrm{d} \tau}{\max \left\{\int \overline{P_{h}}\left(t_{k}, \tau\right)^{2} \mathrm{~d} \tau, \int \overline{P_{h}}\left(t_{k}+\Delta t, \tau\right)^{2} \mathrm{~d} \tau\right\}} .
$$

The stationary interval can be then calculated as

$$
T_{s}\left(t_{k}\right)=\max \left\{\left.\Delta t\right|_{c\left(t_{k}, \Delta t\right) \geq c_{\text {thresh }}}\right\},
$$

where $c_{\text {thresh }}$ is a given threshold of the correlation coefficient. Fig. 12 shows the empirical complementary cumulative distribution functions (CCDFs) of stationary intervals for our proposed HST simulation model and the measured HST channel using the following simulation parameters obtained from [27]: $f_{c}=930 \mathrm{MHz}, v_{R}=324 \mathrm{~km} / \mathrm{h}, N_{P D P}=$ $15, c_{\text {thresh }}=0.8$. It is worth mentioning that since the measured HST channel is a narrowband one, in (43) we used $\tilde{h}_{p q}\left(t_{k}, \tau\right)=\tilde{h}_{1, p q}\left(t_{k}\right) \delta\left(\tau-\tau_{1}\right)$ where $\tilde{h}_{1, p q}\left(t_{k}\right)$ is given in (34). The excellent agreement between the proposed HST simulation model and the measurement data demonstrates the utility of our HST channel models. From Fig. 12, the stationary interval is equal to $11 \mathrm{~ms}$ for $80 \%$ and $20 \mathrm{~ms}$ for $60 \%$ which is considerably shorter than the ones reported for standardized channel models, i.e., $37.8 \mathrm{~ms}$ for $60 \%$ as we previously mentioned in the introduction.

\section{Conclusions}

In this paper, we have proposed a non-stationary theoretical wideband MIMO HST GBSM for the outdoor channel of HST communication systems adopting MRS technology. The proposed model contains time-varying model parameters, i.e., angular parameters and distance between the Tx and Rx. Using the MMEA, a corresponding SoS simulation model 
has been developed. Both models can be further used to model non-stationary V2I channels. The statistical properties of both models have been investigated based on a proposed framework that describes non-stationary channels. Numerical analyses have shown that these statistical properties experience different behaviours at different time instants, demonstrating the capability of the proposed models to mimic the nonstationarity of HST channels. The dominance of the LoS component has resulted in higher correlation in the first tap of the proposed channel models in comparison with the second one. It has also been demonstrated that the time-varying angles will affect the time-variant space CCFs and time-variant ACFs while time-varying dimensions of the ellipses will only have an influence on the time variant ACFs. Moreover, the trends of the time-variant SD PSDs are considerably affected by the angular parameters of the channel models. For all statistical properties, the simulation model provides good approximation to the theoretical one. Finally, it has been shown that the stationary interval of our simulation model matches well that of the measured data, which validates the utility of the proposed model. Our future work will further verify the proposed models by fitting more statistical properties to those of relevant measurement data, which have not yet been available in the existing literature to the best of our knowledge.

\section{REFERENCES}

[1] ETSI website, http://www.etsi.org/technologiesclusters/technologies/intelligent-transport.

[2] D. W. Matolak and Q. Wu, "Vehicle-to-vehicle channels: are we done yet?," in Proc. IEEE GLOBECOM'09, Hawaii, USA, Dec. 2009.

[3] Y. Zhou, Z. Pan, J. Hu, J. Shi, and X. Mo, "Broadband wireless communications on high speed trains," in Proc. IEEE WOCC'11, New Jersey, USA, Apr. 2011.

[4] H. Wu, Y. Gu, and Z. Zhong, "Research on the fast algorithm for GSM$\mathrm{R}$ switching for high-speed railway," Journal of Railway Engineering Society, vol. 124, pp. 92-98, Jan. 2009.

[5] 3GPP, TS36.104, V11.3.1, "3rd Generation Partnership Project; Technical Specification Group Radio Access Network; Evolved Universal Terrestrial Radio Access (E-UTRA); Base Station (BS) radio transmission and reception (Release 11)," Jan. 2013.

[6] O. B. Karimi, L. J. Liu, and C. Wang, "Seamless wireless connectivity for multimedia services in high speed trains," IEEE J. Sel. Areas Commun., vol. 30, no. 4, pp. 729-739, May 2012.

[7] J. Wang, H. Zhu, and N. J. Gomes, "Distributed antenna systems for mobile communications in high speed trains," IEEE J. Sel. Areas Commun., vol. 30, no. 4, pp. 675-683, May 2012.

[8] D. Lee, H. Seo, B. Clerckx, E. Hardouin, D. Mazzarese, S. Nagata, and K. Sayana, "Coordinated multipoint transmission and reception in LTE-advanced: deployment scenarios and operational challenges," IEEE Commun. Mag., vol. 50, no. 2, pp. 148-155, Feb. 2012.

[9] F. Haider, C.-X. Wang, H. Haas, D. Yuan, H. Wang, X. Gao, X.-H. You, and E. Hepsaydir, "Spectral efficiency analysis of mobile femtocell based cellular systems," in Proc. IEEE ICCT'11, Jinan, China, Sept. 2011, pp. 347-351.

[10] ITU-R M.2135-1, "Guidelines for Evaluation of Radio Interface Technologies for IMT-Advanced," Geneva, Switzerland, Rep. ITU-R M.21351, Dec. 2009.

[11] P. Kyösti, et al., "WINNER II channel models," IST-4-027756, WINNER II D1.1.2, v1.2, Apr. 2008.

[12] L. Tain, Y. Zhou, J. Li, Y. Huang, J. Shi, and J. Zhou, "A novel handover scheme for seamless wireless connectivity in high-speed rail," in Proc. IEEE WiMob'11, Shanghai, China, Oct. 2011, pp. 230-236.

[13] W. Dong, G. Liu, L. Yu, H. Ding, and J. Zhang, "Channel properties of indoor part for high-speed train based on wideband channel measurement," in Proc. IEEE CHINACOM'10, Beijing, China, Aug. 2010.

[14] D. W. Matolak, "Channel modeling for vehicle-to-vehicle communications," IEEE Commun. Mag., vol. 46, no. 5, pp. 76-83, May 2008.
[15] C.-X. Wang, X. Hong, X. Ge, X. Cheng, G. Zhang, and J. S. Thompson, "Cooperative MIMO channel models: a survey," IEEE Commun. Mag., vol. 48, no. 2, pp. 80-87, Feb. 2010.

[16] C.-X. Wang, X. Cheng, and D. I. Laurenson, "Vehicle-to-vehicle channel modeling and measurements: recent advances and future challenges," IEEE Commun. Mag., vol. 47, no. 11, pp. 96-103, Nov. 2009.

[17] A. F. Molisch, F. Tufvesson, J. Karedal, and C. F. Mecklenbrauker "A survey on vehicle-to-vehicle propagation channels," IEEE Wireless Commun. Mag., vol. 16, no. 6, pp. 12-22, Dec. 2009.

[18] A. Paier et al. "Non-WSSUS vehicular channel characterization in highway and urban scenarios at $5.2 \mathrm{GHz}$ using the local scattering function," in International ITG Workshop on Smart Antennas, Darmstadt, Germany, Feb. 2008, pp. 9-15.

[19] K. Pekka, "WINNER II channel models part II radio channel measurement and analysis results," IST-4-027756, WINNER II D1.1.2, v1.0, Sep. 2007.

[20] R. Parviainen, P. Kyosti, Y. Hsieh, P. Ting and J. Chiou, "Results of high speed train channel measurements," in COST 2100 TD'08, Lille, France, Oct. 2008.

[21] M. V. S. N. Prasad, K. Ratnamla, and P. K. Dalela, "Communication measurements along railroads and model evaluations over eastern-Indian rural regions," IEEE Antennas and Propagation Magazine, vol. 52, no. 5, pp. 131-141, Oct. 2010.

[22] R. He, Z. Zhong, B. Ai, and J. Ding, "An empirical path loss model and fading analysis for high-speed railway viaduct scenarios," IEEE Antennas and Wireless Propagation Letters, vol. 10, pp. 808-812, Aug. 2011.

[23] L. Liu, C, Tao, J, Qiu, H. Chen, L. Yu, W. Dong, and Y. Yuan, "Positionbased modeling for wireless channel on high-speed railway under a viaduct at $2.35 \mathrm{GHz}$," IEEE J. Sel. Areas Commun., vol. 30, no. 4, pp. 834-845, May 2012.

[24] J. Qiu, C. Tao, L. Liu, and Z. Tan, "Broadband channel measurement for the high-speed railway based on WCDMA," in Proc. IEEE VTC'12Spring, Yokohama, Japan, May 2012.

[25] S. Knorzer, M. A. Baldauf, T. Fugen, and W. Wiesbeck, "Channel analysis for an OFDM-MISO train communications system using different antennas," in Proc. IEEE VTC'07-Fall, Baltimore, USA, Oct. 2007, pp. 809-813.

[26] K. Pahlavan and A. Levesquec, Wireless Information Networks. 2nd ed. NewYork: Wiley, 2005.

[27] B. Chen, Z. Zhong, and B. Ai, "Stationarity intervals of time-variant channel in high speed railway scenario," J. China Commun., vol. 9, no. 8, pp. 64-70, Aug. 2012.

[28] C.-X. Wang, M. Pätzold, and D. Yuan, "Accurate and efficient simulation of multiple uncorrelated Rayleigh fading waveforms," IEEE Trans. Wireless Commun., vol. 6, no. 3, pp. 833-839, Mar. 2007.

[29] C.-X. Wang, X. Hong, H. Wu, and W. Xu, "Spatial temporal correlation properties of the $3 \mathrm{GPP}$ spatial channel model and the Kronecker MIMO channel model," EURASIP J. Wireless Commun. and Networking, vol. 2007, Article ID 39871, 9 pages, 2007. doi:10.1155/2007/39871.

[30] X. Cheng, C.-X. Wang, H. Wang, X. Gao, X.-H. You, D. Yuan, B. Ai, Q. Huo, L. Song, and B. Jiao, "Cooperative MIMO channel modeling and multi-link spatial correlation properties," IEEE J. Sel. Areas Commun., vol. 30, no. 2, pp. 388-396, Feb. 2012.

[31] P. Bello, "Characterization of randomly time-variant linear channels," IEEE Trans. on Wireless Commun., vol. 11, no. 4, pp. 360-393, Dec. 1963.

[32] G. Matz, "On non-WSSUS wireless fading channels," IEEE Trans. on Wireless Commun., vol. 4, no. 5, pp. 2465-2478, Sep. 2005.

[33] A. Chelli and M. Pätzold, "A non-stationary MIMO vehicle-to-vehicle channel model derived from the geometrical street model," in Proc. IEEE VTC'11-Fall, San Francisco, USA, Sept. 2011.

[34] A. Ghazal, C.-X. Wang, H. Haas, M. A. Beach, X. Lu, and D. Yuan, "A non-stationary MIMO channel model for high speed train communication systems," in Proc. IEEE VTC'12-Spring, Yokohama, Japan, May 2012.

[35] A. Ghazal, C.-X. Wang, H. Haas, M. Beach, R. Mesleh, D. Yuan, X. $\mathrm{Ge}$, and M. K. Chahine, "A non-stationary geometry-based stochastic model for MIMO high-speed train channels," invited paper, in Proc. IEEE ITST'12, Taipei, Taiwan, Nov. 2012, pp. 7-11.

[36] R. Kattenbach, "Statistical modeling of small-scale fading in directional radio channels," IEEE J. Sel. Areas Commun., vol. 20, no. 3, pp. 584 592, Apr. 2002

[37] M. Pätzold, "System function and characteristics quantities of spatial deterministic Guassian uncorrelated scattering processes," in Proc. IEEE VTC'03-Spring, Jeju, Korea, Apr. 2003, pp. 256-261.

[38] M. Herdin, "Non-Stationary Indoor MIMO Radio Channels," PhD thesis, Technische Universität Wien, Aug. 2004. 
[39] S. Payami and F. Tufvesson, "Channel measurements and analysis for very large array systems at $2.6 \mathrm{GHz}$," in Proc. IEEE EuCAP'12, Prague, Czech Republic, Mar. 2012, pp. 433-437.

[40] R. Vaughan and J. Bach-Anderson, Channels, Propagation and Antennas for Mobile Communications, London: IET, 2003.

[41] X. Cheng, Q. Yao, M. Wen, C.-X. Wang, L. Song, and B. Jiao, "Wideband channel modeling and ICI cancellation for vehicle-to-vehicle communication systems," IEEE J. Sel. Areas Commun., vol. 31, no. 9, pp. 434-448, Sept. 2013.

[42] M. Pätzold, Mobile Radio Channels. 2nd ed. Chichester: Wiley, 2011.

[43] X. Cheng, C.-X. Wang, D. I. Laurenson, S. Salous, and A. V. Vasilakos, "An adaptive geometry-based stochastic model for non-isotropic MIMO mobile-to-mobile channels," IEEE Trans. Wireless Commun., vol. 8, no. 9 , Sep. 2009, pp. 4824-4835.

[44] Y. Ma and M. Pätzold, "A wideband one-ring MIMO channel model under non-isotropic scattering conditions," in Proc. IEEE VTC'08-Spring, Singapore, May 2008, pp. 424-429.

[45] X. Cheng, C.-X. Wang, and D. I. Laurenson, "Multiple-ring based modeling and simulation of wideband space-time-frequency MIMO channels," in Proc. IEEE ICC'09, Dresden, Germany, June 2009, pp. 4586-4590.

[46] Y. Yuan, A. Ghazal, Q. Yao, and C.-X. Wang, "Non-stationary IMTA MIMO channel model with time-varying AoDs and AoAs," Huawei Project WP3 Internal Report, May 2012.

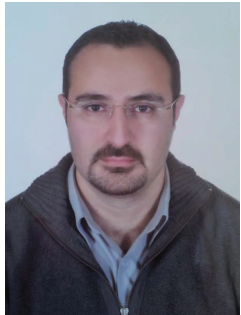

Ammar Ghazal received the BSc degree in Electronics and Telecommunication Engineering from Damascus University, Syria, in 2006 and MSc by Research from Heriot-Watt University, Edinburgh, U.K., in 2010. Since January 2011, he has been a $\mathrm{PhD}$ student at Heriot-Watt University. His main research interests include wireless propagation channel characterization and modeling, non-stationary channel models, and high-speed train wireless communications.

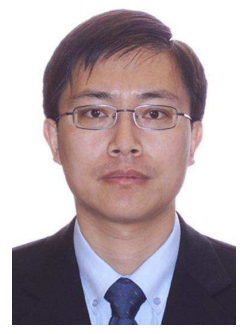

Cheng-Xiang Wang (S'01-M'05-SM'08) received the BSc and MEng degrees in Communication and Information Systems from Shandong University, China, in 1997 and 2000, respectively, and the PhD degree in Wireless Communications from Aalborg University, Denmark, in 2004.

He has been with Heriot-Watt University, Edinburgh, U.K., since 2005 and was promoted to a Professor in 2011. He is also an Honorary Fellow of the University of Edinburgh, U.K., and a Chair/Guest Professor of Shandong University and Southeast University, China. He was a Research Fellow at the University of Agder, Grimstad, Norway, from 2001-2005, a Visiting Researcher at Siemens AGMobile Phones, Munich, Germany, in 2004, and a Research Assistant at Technical University of Hamburg-Harburg, Hamburg, Germany, from 20002001. His current research interests include wireless channel modeling, green communications, cognitive radio networks, vehicular communication networks, massive MIMO, millimeter wave communications, and 5G wireless communication networks. He has edited 1 book and published 1 book chapter and over 200 papers in refereed journals and conference proceedings.

Prof. Wang served or is currently serving as an editor for 8 international journals, including IEEE Transactions on Vehicular Technology (2011-) and IEEE Transactions on Wireless Communications (2007-2009). He was the leading Guest Editor for IEEE Journal on Selected Areas in Communications, Special Issue on Vehicular Communications and Networks. He served or is serving as a TPC member, TPC Chair, and General Chair for over 70 international conferences. He received the Best Paper Awards from IEEE Globecom 2010, IEEE ICCT 2011, ITST 2012, and IEEE VTC 2013-Fall. $\mathrm{He}$ is a Fellow of the IET, a Fellow of the HEA, and a member of EPSRC Peer Review College.

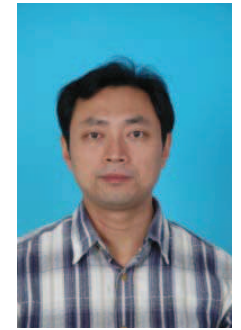

Bo Ai (M'00-SM'09) received his MSc and Ph.D degrees from Xidian University, China, in 2002 and 2004, respectively. He graduated in 2007 with great honors of Excellent Postdoctoral Research Fellow in Tsinghua University. He is now working in Beijing Jiaotong University as a professor and $\mathrm{Ph} . \mathrm{D}$ supervisor. He is a deputy director of State Key Laboratory of Rail Traffic Control and Safety. He is an associate editor for IEEE Trans. on Consumer Electronics and an editorial committee member of Journal of Wireless Personal Communications. He has authored/co-authored 6 books, 26 invention patents, and 130 scientific research papers. His current research interests include OFDM techniques, HPA linearization techniques, radio propagation and channel modeling, and GSM Railway systems. He is a Senior Member of Electronics Institute of China (CIE).

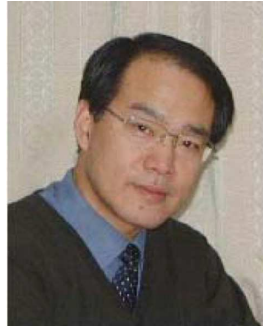

Dongfeng Yuan (SM'01) received the MSc degree in Department of Electrical Engineering, Shandong University, China, in 1988, and got the Ph.D. degree in Department of Electrical Engineering, Tsinghua University, China, in January 2000. Currently, he is a full professor in School of Information Science and Engineering, Shandong University, China. From 1993 to 1994, he was with Electrical and Computer Department at the University of Calgary, Alberta, Canada. He was with Department of Electrical Engineering in the University of Erlangen, Germany from 1998 to 1999, with Department of Electrical Engineering and Computer Science in the University of Michigan, Ann Arbor, USA, from 2001 to 2002, with Department of Electrical Engineering in Munich University of Technology, Germany, in 2005, and with Department of Electrical Engineering in Heriot-Watt University, UK, in 2006. His current research interests include cognitive radio systems, cooperative (relay) communications, and 4G wireless communications.

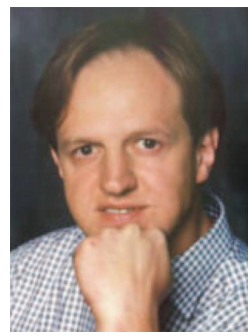

Harald Haas (S'98-AM'00-M'03) holds the Chair of Mobile Communications in the Institute for Digital Communications (IDCOM) at the University of Edinburgh. His main research interests are in the areas of wireless system design and analysis as well as digital signal processing, with a particular focus on interference coordination in wireless networks, spatial modulation and optical wireless communication.

Professor Haas holds more than 15 patents. He has published more than 50 journal papers including a Science Article and more than 140 peer-reviewed conference papers. Nine of his papers are invited papers. He has coauthored a book entitled Next Generation Mobile Access Technologies: Implementing TDD with Cambridge University Press. Since 2007, he has been a Regular High Level Visiting Scientist supported by the Chinese 111 program at Beijing University of Posts and Telecommunications (BUPT). He was an invited speaker at the TED Global conference 2011. He has been shortlisted for the World Technology Award for communications technology (individual) 2011 